\title{
The link between rotavirus vaccination and intussusception: implications for vaccine strategies
}

\author{
C M Cale, N J Klein
}

Strong association between vaccination with RRV-TV and intussusception among otherwise healthy infants supports a causal relationship

B $y$ the age of five years, between 1 in 40 and 1 in 77 children in Europe and the USA will have been hospitalised for rotavirus diarrhoea. ${ }^{1} \mathrm{Al}$ though health care and economic costs of the illness are high, mortality in developed countries is very low. In contrast with the developing world, and despite considerable efforts to educate carers in simple oral rehydration therapy, it is estimated that $600000-800000$ children, or 1 in 40 children in the first five years of life, die annually from rotavirus infection. ${ }^{2}$ The advantages of an oral rotavirus vaccine thus seem self evident: economic benefits to the developed world and significantly reduced infant mortality in the developing world. Candidate rotavirus vaccines were first developed by tissue culture adaptation and attenuation of bovine and rhesus rotaviruses. Subsequently, such heterologous rotaviruses were improved for use as human vaccines by reassortment with human rotaviruses. After favourable results of prelicensing trials in the USA and Finland ${ }^{3-6}$ showing vaccine efficacies of $68 \%$ and $91 \%$ for reduction of all and serious rotavirus infections, respectively, the US food and drug administration licensed an oral tetravalent rhesushuman reassortant rotavirus vaccine (RRV-TV) in August 1998. Distribution of the vaccine for incorporation into infant schedules commenced in the USA in October 1998. By the end of May 1999, when approximately 1.5 million doses of RRV-TV vaccine had been distributed, nine cases of intussusception in infants who had received RRV-TV had been reported to the Vaccine Adverse Events Reporting System, as compared with only four reports to that organisation in the preceding seven years. ${ }^{7}$ As a result of these data, the vaccination programme was initially suspended and the company withdrew the vaccine from the market in October 1999. Three questions arise as a result of these actions:

-was this a true effect?

-what was the mechanism of the effect? -what are the ethical implications?

The first of these questions was carefully addressed in the recently published investigation by the Rotavirus Intussusception Investigation team of the Centres for Disease Control (CDC) in

Murphy TV, Gargiullo PM, Massoudi MS, et al. Intussusception among infants given an oral rotavirus vaccine. N Engl J Med 2001;344:564-72.

Background: Intussusception is a form of intestinal obstruction in which a segment of the bowel prolapses into a more distal segment. Our investigation began on May 27, 1999, after nine cases of infants who had intussusception after receiving the tetravalent rhesus-human reassortant rotavirus vaccine (RRV-TV) were reported to the Vaccine Adverse Event Reporting System.

Methods: In 19 states, we assessed the potential association between RRV-TV and intussusception among infants at least 1 but less than 12 months old. Infants hospitalized between November 1, 1998, and June 30, 1999, were identified by systematic reviews of medical and radiologic records. Each infant with intussusception was matched according to age with four healthy control infants who had been born at the same hospital as the infant with intussusception. Information on vaccinations was verified by the provider

Results: Data were analyzed for 429 infants with intussusception and 1763 matched controls in a case-control analysis as well as for 432 infants with intussusception in a case-series analysis. Seventy-four of the 429 infants with intussusception (17.2 percent) and 226 of the 1763 controls (12.8 percent) had received RRV-TV ( $P=0.02)$. An increased risk of intussusception 3 to 14 days after the first dose of RRV-TV was found in the case-control analysis (adjusted odds ratio, 21.7; 95 percent confidence interval, 9.6 to 48.9). In the case-series analysis, the incidence-rate ratio was 29.4 (95 percent confidence interval, 16.1 to 53.6) for days 3 through 14 after a first dose. There was also an increase in the risk of intussusception after the second dose of the vaccine, but it was smaller than the increase in risk after the first dose. Assuming full implementation of a national program of vaccination with RRV-TV, we estimated that 1 case of intussusception attributable to the vaccine would occur for every 4670 to 9474 infants vaccinated.

Conclusions: The strong association between vaccination with RRV-TV and intussusception among otherwise healthy infants supports the existence of a causal relation. Rotavirus vaccines with an improved safety profile are urgently needed.

the USA. ${ }^{7}$ The study was based on retrospective case controlled and case series analyses of infants aged 1-12 months, hospitalised with radiologically or operatively confirmed intussusception between 1 November 1998 and 30 June 1999 across the 19 states of the USA where $80 \%$ of the administered RRV-TV had been distributed. Of the 446 infants identified with intussusception, 429 were included in the study (96\%). A total of $17.2 \%$ of the patients with intussusception and $12.8 \%$ of the controls had received RRV-TV $(\mathrm{p}=0.02)$. The severity of intussusception was not significantly different in control and vaccinated patients. In the RRV-TV group, the risk of intussusception was greatest in the 3-14 days after any dose of vaccine, with an odds ratio of 10.6. This translated to an odds ratio of 21.7 after the first dose when the majority $(64 \%)$ of cases occurred. The authors estimated that if the
RRV-TV programme were fully implemented across the USA, between 4670 and 9474 infants would be vaccinated for each case of intussusception attributable to RRV-TV. They concluded that their data provided evidence of a strong temporal and specific link between RRV-TV and intussusception.

The CDC's retrospective study was not designed to address the aetiology of the link between RRV-TV vaccine and intussusception. Indeed, the pathogenesis of intussusception in infants is poorly understood. The observation of mesenteric lymphadenopathy or inflamed Peyer's patches in a significant proportion of cases points to an inflammatory process. This has been linked to a number of viral infections: commonly adenovirus, but also human herpes virus (HHV)-6, HHV-7, Ebstein-Barr virus, and cytomegalovirus. ${ }^{8}$ Although uncommon, wild rotavirus infection has also been 
identified in infants with intussusception. ${ }^{9}$ The incidence of this complication may be serotype dependent, ${ }^{10}$ possibly because some serotype specific rotaviral enterotoxins can cause lymphoid hyperplasia and increased intestinal peristalsis. ${ }^{11}$ This clearly has implications for the selection of serotypes in novel rotavirus vaccines.

The third question raised by this vaccine experience is an ethical one. Withdrawal of RRV-TV by the manufacturer has huge implications for the developed world, where the benefits in terms of reduction in infant mortality are likely to be most significant. To date, the only trials of the vaccine outside Europe and the USA have been in South America where reduced vaccine efficacies were observed. ${ }^{12}$ This may be related to the prevalent rotavirus serotypes in South America, which differ from those in the USA and Europe. ${ }^{12}$ Information on the incidence of intussusception in this study population is not available. No studies have been undertaken in Africa, which has a similar serotype distribution to the USA and Europe, ${ }^{13}$ and thus where one might predict similarly good vaccine efficacy. While the risk-benefit analysis for RRV-TV and related vaccines may be favourable in some parts of the world, withdrawal of one rotavirus vaccine is likely to make pharmaceutical companies wary of pursuing current or future rotavirus vaccine developments.

There is little doubt of the worldwide need for an effective and safe rotavirus vaccine. However, without a full understanding of the pathophysiology of intestinal intussusception, it will be difficult to design vaccines that avoid this complication.

Gut 2002;50:1 1-12

\section{Authors' affiliations}

C M Cale, Department of Immunology, Great Ormond Street Hospital for Children NHS Trust, London, UK

N J Klein, Immunobiology Unit, Institute of Child Health, London, UK

Correspondence to: $\operatorname{Dr} C M$ Cale, Immunology Department, Camelia Botnar Laboratories, Great Ormond Street Hospital for Children NHS Trust, Great Ormond Street, London WCiN 3JH, UK. calec@gosh.nhs.uk

\section{REFERENCES}

1 Glass RI, Bresee JS, Parashar UD, et al. First rotavirus vaccine licensed: is there really a need? Acta Pediatr Suppl 1999;88:2-8.

2 Bern C, Martines J, de Zoysa I, et al. The magnitude of the global problem of diarrhoeal disease: a ten year update. Bull WHO 1992;70:705-14.
3 Rennels MD Glass RI, Dennehy PH, et al. Safety and efficacy of high-dose tetravalent reassortant vaccines - report of a National Multicentre trial. Pediatrics 1996;97:7-13

4 Bernstein DI, Glass RI, Rogers G, et al. Evaluation of rhesus rotavirus monovalent and tetravalent reassortant vaccines in US children. JAMA 1996;273:1191-6.

5 Joensuu J, Koskenniemi E, Pang XL, et al. Randomised placebo-controlled trial of rhesus-human reassortant rotavirus vaccine for prevention of severe rotavirus gastroenteritis. Lancet 1997:350:1205-9.

6 Santosham M, Moulton LH, Reid R, et al. Efficacy and safety of high-dose rhesus-human reassortant vaccine in Native American populations. J Pediatr 1997:131:632-8.

7 Murphy TV, Garguillo PM, Massoudi MS, et al. Intussusception among infants given an oral rotavirus vaccine. N Engl I Med 2001;344:564-72.

8 Hsu HY, Kao CL, Huang LM, et al. Viral etiology of intussusception in Taiwanese childhood. Pediatr Infect Dis J 1998:17:893-8.

9 Konno T, Suzuki H, Kutsuzawa, et al. Human rotavirus infection in infants and young children with intussusception. J Med Virol 1978;2:265-9.

10 Nakagomi T. Rotavirus infection and intussusception: a view from retrospect. Microbiol Immunol 2000;44:619-28.

11 Lundgren O, Peregrin AT, Persson K, et al. Role of the enteric nervous system in the fluid and electrolyte secretion of rotavirus diarrhea. Science 2000;287:491-5.

12 Linhares AC, Bresee JS. Rotavirus vaccines and vaccination in Latin America. Rev Panam Salud Publica 2000;8:305-31.

13 Cunliffe NA, Kilgore PE, Bressee JS, et al. Epidemiology of rotavirus diarrhoea in Africa: a review to assess the need for rotavirus immunisation. Bull WHO 1998;76:525-37. 